\title{
Electron localization and optical absorption of polygonal quantum rings
}

\author{
Anna Sitek* \\ Science Institute, University of Iceland, Dunhaga 3, IS-107 Reykjavik, Iceland \\ and Department of Theoretical Physics, Wroctaw University of Technology, 50-370 Wroctaw, Poland \\ Llorenç Serra \\ Institute of Interdisciplinary Physics and Complex Systems IFISC (CSIC-UIB), \\ and Department of Physics, University of the Balearic Islands, E-07122 Palma de Mallorca, Spain \\ Vidar Gudmundsson \\ Science Institute, University of Iceland, Dunhaga 3, IS-107 Reykjavik, Iceland \\ Andrei Manolescu \\ School of Science and Engineering, Reykjavik University, Menntavegur 1, IS-101 Reykjavik, Iceland
}

(Received 31 March 2015; revised manuscript received 28 May 2015; published 17 June 2015)

\begin{abstract}
We investigate theoretically polygonal quantum rings and focus mostly on the triangular geometry where the corner effects are maximal. Such rings can be seen as short core-shell nanowires, a generation of semiconductor heterostructures with multiple applications. We show how the geometry of the sample determines the electronic energy spectrum, and also the localization of electrons, with effects on the optical absorption. In particular, we show that irrespective of the ring shape low-energy electrons are always attracted by corners and are localized in their vicinity. The absorption spectrum in the presence of a magnetic field shows only two peaks within the corner-localized state domain, each associated with different circular polarization. This picture may be changed by an external electric field which allows previously forbidden transitions, and thus enables the number of corners to be determined. We show that polygonal quantum rings allow absorption of waves from distant ranges of the electromagnetic spectrum within one sample.
\end{abstract}

DOI: 10.1103/PhysRevB.91.235429

PACS number(s): 73.21.La, 73.22.Dj, 78.67.Hc

\section{INTRODUCTION}

Recently it has become feasible to grow core-multiple-shell nanowires consisting of a core built of one type of material which is surrounded by one or more shells of different materials. This preparation method makes achievable a huge variety of heterostructures with various and controllable properties which make them extremely attractive as building blocks of nanoelectronic and optoelectronic nanodevices, in particular solar cells [1-4] or nanoantennas [5]. In particular, nanowires of triangular cross section turned out to be a very good host for robust and efficient coaxial $p-i-n$ junctions [6] or multicolor nanophotonic sources with controllable wavelengths [7-10]. Besides these applications, we would also like to mention a basic theoretical interest in polygonal rings as particular examples of quantum graphs [11] with characteristic physical behaviors. Such nanowires are usually grown vertically and, due to the crystallographic structure, have polygonal cross sections, most commonly hexagonal [12-14], but triangular $[6,8,9,15]$, square [16,17], and dodecagonal [18] cross sections are also feasible. Sharp edges along the wires induce unique carrier localization, which leads to formation of one-dimensional (1D) channels in corner or side areas [19-25].

Core-shell structures allow for modeling of many properties including band alignment, which strongly depends on the strain in the system and may be controlled through the core

\footnotetext{
*sitek@hi.is
}

and/or shell thickness [26]. In such a way one may grow systems in which electrons are confined only in the shell area [12]. It is also possible to etch the core part and achieve hollow nanowires $[13,14]$, i.e., nanotubes of finite thickness. Multishell structures allow growth of narrow (up to $1.5 \mathrm{~nm}$ ) tubes which are formed between two shell layers such that surface effects are reduced [19,24,25]. A polygonal nanoring may be considered as a short wire of this kind.

Some insight has already been gained for hexagonal quantum rings which due to their symmetry and the possibility to localize electrons in the corners are refereed to as artificial benzene [27]. Electron localization at the corners of a polygonal quantum ring is expected if one notes that localization occurs whenever a nanowire is bent. Indeed, electronic states on nanowire bends, which in our case are the corners of the polygonal contour, attracted much interest some years ago [28-35]. In a single-mode wire with a circular bend a simplified 1D picture was obtained in which the corner may be replaced by a square well, whose depth and length are determined by the angle and radius of the circular bend [30]. This approach was used in Ref. [36] to suggest a scattering model of $1 \mathrm{D}$ polygonal wires, treating each corner as a scatterer. However, in contrast to our present purpose, the authors of Ref. [36] considered only the extended states along the sides of the $1 \mathrm{D}$ polygon. In fact, contrary to hexagonal quantum dot molecules, rings also allow localization of charge carriers in side areas, and they can even favor one of the sides if that is sufficiently thick [27]. Other related effects, like the suppression of the Aharonov-Bohm effect in hexagonal rings, have also been theoretically envisioned [37]. 
In this paper we study electron localization in polygonal quantum rings of various shapes and show how it determines optical absorption. We use a computational method based on finite differences on a polar grid which enables us to model not only hexagonal structures but arbitrary polygonal rings including nonsymmetric samples. We also derive the localized states with a $1 \mathrm{D}$ scattering model. We focus mostly on triangular rings, where the corner-to-edge ratio is largest, resulting in the most pronounced localization and corresponding optical effects. We show that irrespective of sample shape one can always distinguish a group of corner-localized states which for some geometries are separated by an energy gap from the higher states. The electron localization pattern is very sensitive to sample symmetry and shape. For quantum rings defined by regular polygonal constraints the localization probability is equally distributed between all corners and/or sides of the ring, but when the symmetry is broken, e.g., by different side thicknesses or corner softening, the probability density becomes localized on individual corners which are occupied according to their areas. External electric fields may partially control electron localization, by destroying equal distribution between corners, or by delocalizing states previously occupying a single corner area.

We analyze optical absorption of the systems and show that in the presence of a perpendicular magnetic field only two transitions occur from the ground state to cornerand side-localized domains, each associated with different polarization. Still, external electric fields may break the wave function symmetry such that more transitions become visible, and thus optical experiments may allow to infer the number of corners. Moreover, we point out that triangular quantum rings allow absorption in the microwave and near-infrared regimes to be observed within the same sample.

The paper is organized as follows. In Sec. II we define the analyzed systems and make a preliminary inspection of the low-energy states in polygonal rings based on the 1D scattering model. Then, in Sec. III we introduce the sample model and describe the discretization method. In Sec. IV we present the low-energy quantum states resulting from our Hamiltonian model. Then in Sec. V we calculate optical spectra corresponding to excitation of electrons initially in the ground state. Finally, Sec. VI contains conclusions and final remarks.

\section{THE 1D SCATTERING MODEL}

The systems under study are two-dimensional (2D) polygonal quantum rings of different shapes. They may also be considered as short core-multiple-shell or hollow nanowires such that all electronic wave functions include only the lowest axial mode. The first part of our analysis is based on a 1D scattering model. A circular bend in a nanowire with a single transverse mode acts approximately like a 1D square-well potential of depth $V_{0}$ and length $2 a$ [30]. The bend radius $R$ and angle $2 \theta$ determine the effective square-well potential through the expressions $V_{0} \simeq-\hbar^{2} /\left(8 m R^{2}\right)$ and $a \simeq R \theta$. Such a potential always supports bound states that, physically, represent states localized on the wire bend [28-35]. This type of effective confinement is the physical mechanism behind the corner localization that occurs in polygonal nanorings of finite width, which will be discussed in detail in Sec. IV.

It is possible to devise a 1D model, whose only coordinate is the position along the nanoring perimeter, say $\xi$, with cyclic boundary conditions on the wave function $\psi(\xi)$; namely, $\psi(0)=\psi(L)$, where $L$ is the full perimeter length. Each corner acts like a point scatterer, characterized by a scattering matrix given by a square well. Using this 1D model the authors of Ref. [36] described the states propagating along the polygon sides. Here we extend that analysis to negative-energy states, localized on the corners and behaving as evanescent waves on the polygon sides.

The wave function between vertices $i$ and $i+1$ is a combination of right $(r)$ and left $(l)$ propagating plane waves,

$$
\begin{aligned}
\psi(\xi) & =a_{r}^{(i)} e^{-i p\left(\xi-\xi_{i}\right)}+b_{r}^{(i)} e^{i p\left(\xi-\xi_{i}\right)} \\
& =a_{l}^{(i+1)} e^{i p\left(\xi-\xi_{i+1}\right)}+b_{l}^{(i+1)} e^{-i p\left(\xi-\xi_{i+1}\right)},
\end{aligned}
$$

where $i=1, \ldots, N_{v}$ label the vertices, $\xi_{i}$ are their positions, and the wave number $p$ is purely imaginary. For a total energy $E$ lower than the first transverse mode of the wire $\epsilon_{1}$ it is $p=i \sqrt{2 m\left(\epsilon_{1}-E\right)} / \hbar$. The scattering amplitudes in Eq. (1) fulfill a linear homogeneous system given by the scattering relation

$$
\left(\begin{array}{l}
b_{l}^{(i)} \\
b_{r}^{(i)}
\end{array}\right)=\left(\begin{array}{ll}
r & t \\
t & r
\end{array}\right)\left(\begin{array}{l}
a_{l}^{(i)} \\
a_{r}^{(i)}
\end{array}\right),
$$

and the condition between successive vertices

$$
\begin{aligned}
& b_{l}^{(i)}=a_{r}^{(i-1)} e^{-i p \ell}, \\
& b_{r}^{(i)}=a_{l}^{(i+1)} e^{-i p \ell} .
\end{aligned}
$$

In Eq. (2) $r$ and $t$ are the reflection and transmission scattering amplitudes of the above-mentioned squared well for imaginary wave numbers. The energies $E$ for which Eqs. (2), (3), and (4) admit a solution can be determined from the zeros of the determinant of the linear system matrix $M$, or equivalently of the function [36]

$$
\mathcal{F}=\operatorname{norm}\left\{\tilde{M}\left(\begin{array}{l}
a \\
b
\end{array}\right)\right\},
$$

where $\tilde{M}$ is analogous to $M$ except for an arbitrarily chosen scattering amplitude which is set to 1 .

Figure 1 shows the energy dependence of $\mathcal{F}$ for a triangular, a square, and a hexagonal sample. The sequence of allowed energies in each polygonal nanoring is seen from the $\mathcal{F}$ zeros while the figure insets show the corresponding 1D densities for each mode (labeled as $N_{v}$ a, $N_{v}$ b, etc.). The probability densities are concentrated on the corners and the modes can be classified into two types: translational symmetric modes (TSM's) having the same density on each segment of the polygon and translational asymmetric modes (TAM's) for which the sides look different. The TAM's (3b, 4b, 6b, 6c) are degenerate, since inversion from the central $\xi$ point leads to another valid solution. Counting also the spin, the degeneracy factors become 2 for symmetric modes and 4 for asymmetric ones.

A closer look at the TSM's of Fig. 1 reveals that there are two types, depending on the density at each side midpoint. 


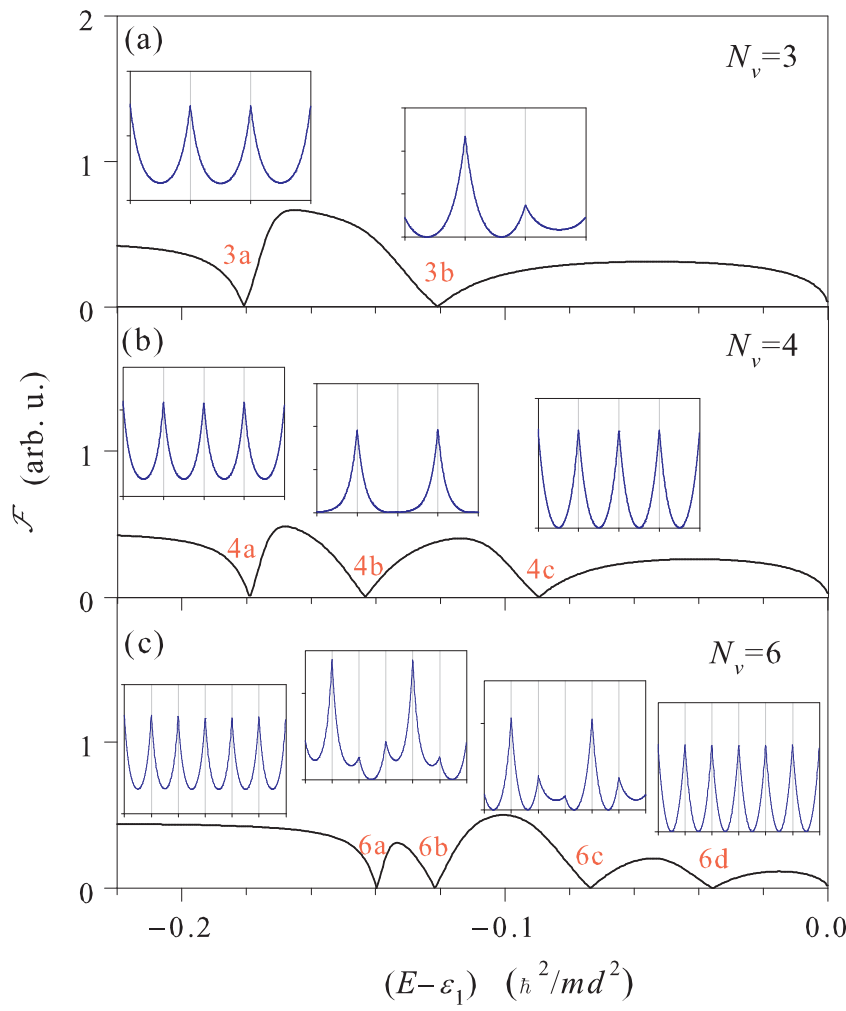

FIG. 1. (Color online) $\mathcal{F}$ function of the 1D model whose zeros signal the allowed states. The insets show the corresponding 1D densities for each zero $\mathcal{F}$ with the vertical lines corresponding to the vertex positions. The number of vertices $N_{v}$ is indicated in each panel. In terms of the width $d$, the side length is fixed at $\ell=5 d$ and the bend radii are $R=0.15 d, 0.1 d$, and $0.07 d$ for the triangle, square, and hexagon, respectively.

Modes 3a, 4a, and 6a have finite midpoint densities while modes $4 \mathrm{c}$ and $6 \mathrm{~d}$ exactly vanish at midpoints. With a similar analysis as that of Ref. [36] it can be shown that the first type $(3 \mathrm{a}, 4 \mathrm{a}, 6 \mathrm{a})$ occurs when $t+r=e^{-i p l}$. The second type of TSM's (4c,6d) correspond to the condition $t+r=-e^{-i p l}$ and occur only in even $N_{v}$ polygons. We also notice that the energies of the TAM's lie in between the TSM's. The sequence is such that in odd- $N_{v}$ polygons there are $N_{v}-1$ TAM's between TSM's, while in even- $N_{v}$ polygons there are $N_{v} / 2-1$ intermediate TAM's. In all cases, however, this sequence of localized states abruptly terminates at the side-propagating threshold $E=\epsilon_{1}$.

\section{THE HAMILTONIAN MODEL}

The second part of our modeling is based on a discretization method on a polar grid. We start with a circular disk geometry as in Ref. [38] on which we apply polygonal constraints and pick up only points within the resulting shell (Fig. 2). In this case the Hilbert space is spanned by vectors $|k j \sigma\rangle$, where $k$ and $j$ label the discretized radial and angular coordinates $\left(r_{k}, \phi_{j}\right)$, with meshes $(\delta r, \delta \phi)$, respectively, and $\sigma$ stands for the two possible spin values.

The system Hamiltonian consists of four terms,

$$
H=H^{K}+H^{E}+H^{B}+H^{Z} .
$$

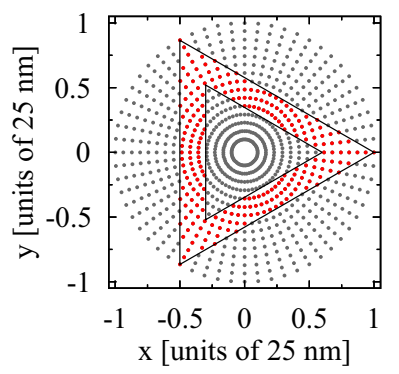

FIG. 2. (Color online) Sample model: Polygonal constraints applied on a polar grid. For visibility we reduced the number of site points.

Hamiltonian matrix elements of the first contribution, the kinetic Hamiltonian, in the $k, j$, and $\sigma$ basis are

$$
\begin{aligned}
H_{k j \sigma, k^{\prime} j^{\prime} \sigma^{\prime}}^{K}= & T \delta_{\sigma, \sigma^{\prime}}\left[t_{r}\left(\delta_{k, k^{\prime}}-\delta_{k, k^{\prime}+1}\right) \delta_{j, j^{\prime}}\right. \\
& \left.+t_{\phi} \delta_{k, k^{\prime}}\left(\delta_{j, j^{\prime}}-\delta_{j, j^{\prime}+1}\right)+\text { H.c. }\right],
\end{aligned}
$$

where $T=\hbar^{2} /\left(2 m^{*} R_{\text {ext }}^{2}\right)$ is a reference energy, $m^{*}$ is the effective mass of the semiconductor material, $R_{\text {ext }}$ is the external radius of the polar grid, $t_{r}=\left(R_{\mathrm{ext}} / \delta r\right)^{2}$, and $t_{\phi}=$ $\left[R_{\text {ext }} /\left(r_{k} \delta \phi\right)\right]^{2}$.

We expose the rings to external electric and magnetic fields. The electric field is parallel to the $x-y$ plane and forms an angle $\varphi$ with the $x$ axis, $\boldsymbol{E}=E(\cos \varphi, \sin \varphi, 0)$, and the corresponding Hamiltonian matrix elements are

$$
H_{k j \sigma, k^{\prime} j^{\prime} \sigma^{\prime}}^{E}=-e \boldsymbol{E} \cdot \boldsymbol{r}_{k} \delta_{k, k^{\prime}} \delta_{j, j^{\prime}} \delta_{\sigma, \sigma^{\prime}},
$$

where $e$ is the electron charge. The magnetic field $\boldsymbol{B}$ is assumed perpendicular to the ring plane, with a vector potential $\boldsymbol{A}=B(-y, x, 0) / 2$, and the corresponding Hamiltonian matrix elements are obtained as

$$
\begin{aligned}
H_{k j \sigma, k^{\prime} j^{\prime} \sigma^{\prime}}^{B}= & T \delta_{\sigma, \sigma^{\prime}} \delta_{k, k^{\prime}} \\
& \times\left[\frac{1}{2} t_{\mathrm{B}}^{2}\left(\frac{r_{k}}{4 R_{\mathrm{ext}}}\right)^{2} \delta_{j, j^{\prime}}-t_{\mathrm{B}} \frac{i}{4 \delta \phi} \delta_{j, j^{\prime}+1}+\text { H.c. }\right],
\end{aligned}
$$

with $t_{\mathrm{B}}=\hbar e B / m^{*} T$ the cyclotron energy in units of $T$.

The last contribution to the Hamiltonian, the Zeeman part, is diagonal in the $k, j$, and $\sigma$ basis

$$
H_{k j \sigma, k^{\prime} j^{\prime} \sigma^{\prime}}^{\mathrm{Z}}=\frac{1}{2} T t_{\mathrm{B}} \gamma\left(\sigma_{z}\right)_{\sigma, \sigma^{\prime}} \delta_{k, k^{\prime}} \delta_{j, j^{\prime}},
$$

where $\gamma=g^{*} m^{*} / 2 m_{e}$ is the ratio between the Zeeman gap and the cyclotron energy, and $m_{e}$ is the free-electron mass. Our discretization method is a version of the very popular hopping schemes used in the mesoscopic physics. Other theoretical studies of core-shell polygonal systems used the finite-element method [23,24,39].

\section{ELECTRONIC STATES}

Below we present results for $2 \mathrm{D}$ polygonal rings achieved with the discretization method where the sample consists of over 6000 grid points. We use the external radius $R_{\text {ext }}=$ $25 \mathrm{~nm}$. We perform numerical calculations for InAs parameters which are $m^{*}=0.023 m_{e}$, where $m_{e}$ is the electron mass, and $g^{*}=-14.9$; thus the energy unit $T$ introduced in the 


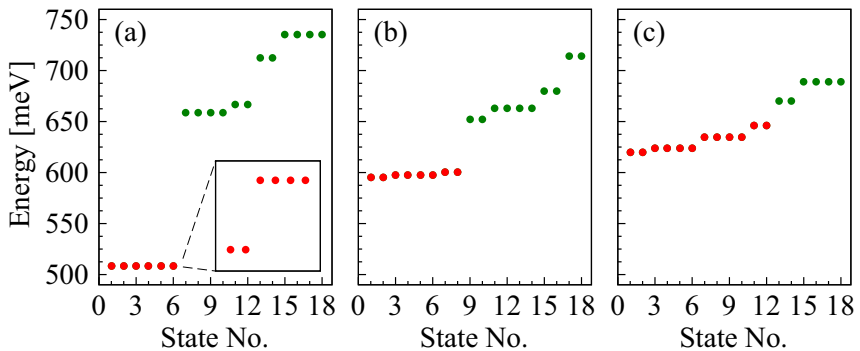

FIG. 3. (Color online) Energy levels for symmetric triangular (a), square (b), and hexagonal (c) samples, of external radii equal to $25 \mathrm{~nm}$ and side thicknesses $5 \mathrm{~nm}$. The inset to (a) shows degeneracy of the two lowest energy levels of the triangular sample. Red points indicate purely corner-localized states.

Hamiltonian (7) equals approximately $2.8 \mathrm{meV}$ and the ratio $\gamma=-0.171$.

\section{A. Symmetric samples}

Symmetric polygonal samples which are restricted externally and internally by regular polygons have well-defined symmetries which imply specific energy degeneracies. In Fig. 3 we compare the energy levels of a single electron confined in symmetric triangular, square, and hexagonal rings, all having sharp corners and $5 \mathrm{~nm}$ side thicknesses, in the absence of external fields. As can be seen, the ground-state energy increases with the number of corners. This is because the size of the effective well formed in the corner area decreases with increasing corner angle, and thus ground-state electrons bounded in $2 \pi / 3$ corners of the regular hexagon have higher energy than those trapped in $\pi / 3$ corners of regular triangles. This is in a nice qualitative agreement with the results shown in Fig. 1. In a circular nanoring the ground state has zero angular momentum and it is doubly (spin) degenerate, whereas all higher states are fourfold degenerate, having finite angular momenta that do not distinguish energetically between clockwise and counterclockwise electron rotations [40-42]. When the regular $N_{v}$ polygonal constraints are applied to a ring structure they break the circular degeneracy at levels corresponding to multiples of $2 N_{v}$. The resulting series of two- and fourfold degenerate energy levels agree with the expectation from Sec. II where spin was ignored.

In Fig. 3(a) a group of the six lowest states of the triangular ring is separated from the higher states. The energy gap behind the eighth state is still visible for a square polygon [Fig. 3(b)], but considerably decreased with respect to the triangular sample, and it practically vanishes for a hexagonal ring [Fig. 3(c)]. Although the energy spacing between the 12th and 13th states of the artificial benzene is comparable with other energy differences, in this case also the lowest states have different character from the others. States associated with the lowest energy levels of symmetric polygonal rings (red points in Fig. 3) are equally distributed between all of the corners, as is shown for a triangular ring in Figs. 4(a), and 4(b). Due to the spin degeneracy the number of these states equals double the number of corners $\left(2 N_{v}\right)$. If the sample is thick enough and contains a sufficient number of corners the probability distribution does not vanish completely in the

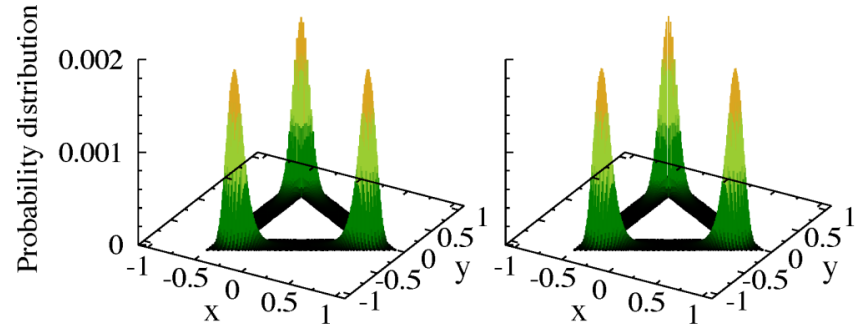

(a) States No. 1, and 2.

(b) States No. 3, 4, 5, and 6.

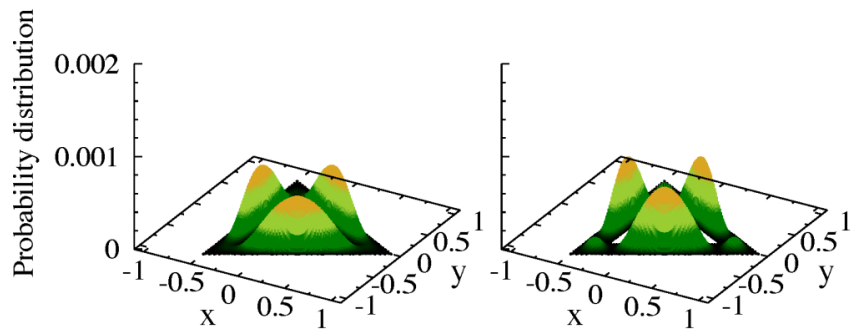

(c) States No. 7, 8, 9, and 10. (d) States No. 11, and 12.

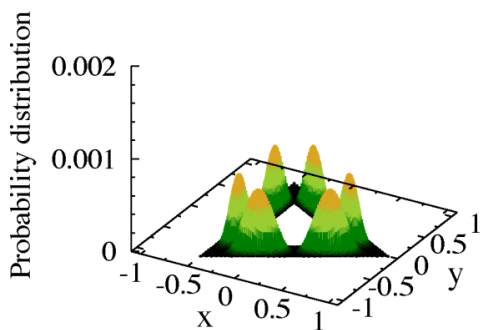

(e) States No. 13, and 14.

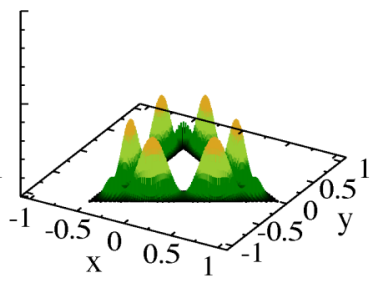

(f) States No. 15, 16, 17, and 18
FIG. 4. (Color online) Probability distribution for the 18 lowest states of a symmetric triangular ring. In (a) and (b) we show localization of the lowest states indicated in red in Fig. 3(a), and in (c), (d), (e), and (f) localization of the states above the energy gap in Fig. 3(a).

middle of the sides, as for the triangular ring shown in Fig. 4, but stabilizes at a much lower level than the corner maxima. The first state above the corner states is purely localized in sides, with maximal probability of finding a particle in the middle of each side [Fig. 4(c)]; higher-energy electrons are also mostly localized in the side areas with only a small probability of finding them in corners [Fig. 4(d)]. The number of probability maxima in the side regions increases with energy and the possibility of finding them in corners becomes relevant [Figs. 4(e), and 4(f)]. The probability of finding electrons in sharp corners becomes comparable to or even exceeds side maxima for high-energy electrons, but the detailed analysis of such states is beyond the scope of this paper.

For the lowest, corner-localized states, the probability density maxima decrease with increasing number of corners and at the same time the density of localization areas increases; similarly, for the first state above them the number of maxima increases and the distances between them decrease with an increasing number of corners, i.e., the side-localization areas decrease. As a result the probability distributions for cornerand side-localized states become relatively similar and thus the energy gap occurring for triangular and square quantum rings [Figs. 3(a) and 3(b)] vanishes for sufficiently thick 


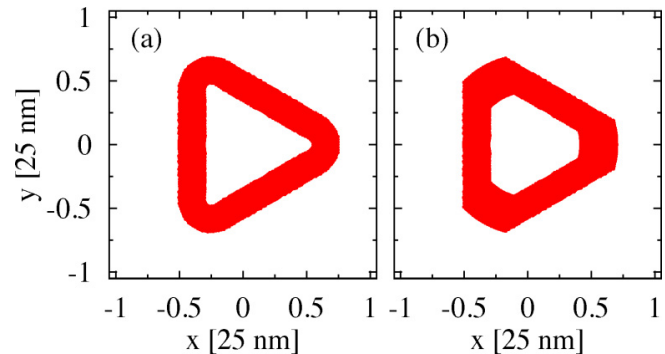

FIG. 5. (Color online) Triangular quantum rings with softened corners. (a) Round corners softened by circles of $r_{\text {int }}=0.05 R_{\text {ext }}$ and $r_{\text {ext }}=0.25 R_{\text {ext }}$ inscribed in the internal and external limiting polygon corners. (b) Corners softened by background ring radii reduced to $70 \%$ of the distance from the center of the sample to the sharp internal and external corners.

hexagonal samples [Fig. 3(c)]. However, the corner-localized states of artificial benzene may also be energetically separated from the higher states when the rings are very narrow such that the corner-localization areas are much smaller than the side ones.

In practice it may be difficult to achieve samples with perfectly sharp corners. Therefore we investigated the impact of corner softening on energy levels and carrier localizations. We analyze two types of symmetric triangular samples shown in Fig. 5, in one case we inscribe circles in the corners which define new internal and external limits in corner areas [Fig. 5(a)]; in the other case we soften corners by cutting the sharp parts by background radii [Fig. 5(b)]. In both cases energy levels show the same degeneracies as for the samples with sharp corners [Fig. 3(a)]. Moreover, the lowest six states associated with the two lowest energy levels are always localized in corner areas. If all of the corners are equally softened and when the softening is relatively small, which for 5-nm-thick samples means that the radii reduction for the sample shown in Fig. 5(b) must be up to around 80\%, then the probability density for samples with soft corners does not differ considerably from the one shown in Fig. 4. There are many possibilities of softening internal and external corners separately; thus there is a huge variety of samples which show properties of ideal (sharp) ones. Interesting features appear when these limits are exceeded. In the case of the sample shown in Fig. 5(a) the energy gap separating the purely corner-localized states is comparable with energy splittings occurring in the next higher states. Those states can be distributed both in the corners and on the sides [Figs. 6(a) and $6(b)]$.

The energy separating the two lowest-energy levels exists in samples with cut corners, like the one shown in Fig. 5(b), but it decreases due to softening. In contrast to the samples with sharp corners, where the lowest six states are localized in the corners and the next six (7th to 13th) states are localized on the sides of the triangle (Fig. 4), now the states associated with the two levels above the energy gap (7th to 13th states) are still localized in the corner area, but each one has two nearby maxima. In fact corner softening of this kind increases the number of corners, and the sample may show mixed features of triangles and hexagons. Three corner maxima split in each

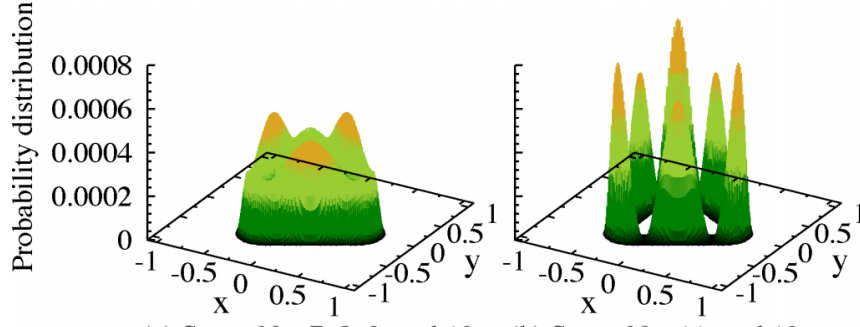

(a) States No. 7, 8, 9, and 10. (b) States No. 11, and 12 .

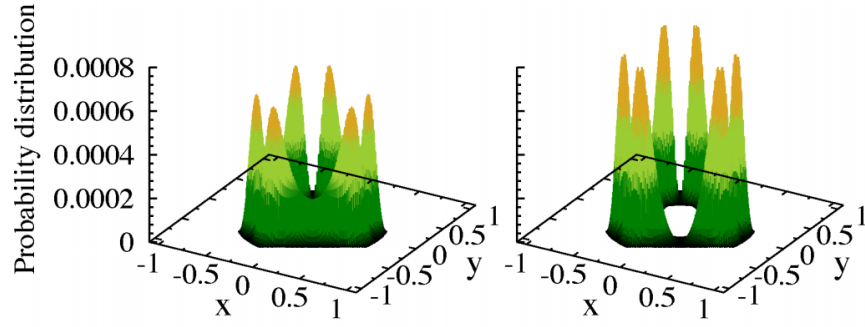

(c) States No. 7, 8, 9, and 10. (d) States No. 11, and 12.

FIG. 6. (Color online) Probability distribution for the states associated with the third (a) and (c) and fourth (b) and (d) energy levels of the soft-corner samples. (a) and (b) refer to the sample shown in Fig. 5(a), and (c) and (d) to the sample in Fig. 5(b).

corner area such that six maxima are formed [Figs. 6(c) and 6(d)] and the transition to mostly (not purely) side-localized states occurs above the fourth energy level as for hexagonal samples.

\section{B. Nonsymmetric samples}

Although the present state of the art of manufacturing allows high-precision control at the single-atom level, it is still difficult to grow perfectly symmetric nanowires, and thus we also analyze different nonsymmetric samples. First we break the symmetry by increasing the thickness of two sides by $5 \%$ and $10 \%$. In this case the energy levels are only spin degenerate and the energy gap between the sixth and seventh states is reduced with respect to the symmetric case, but it is still relevant (Fig. 7). The lowest states are also localized in corner areas (red points in Fig. 7), but the probability distribution is not spread on all corners as before: electrons of specific energy values occupy only one corner.

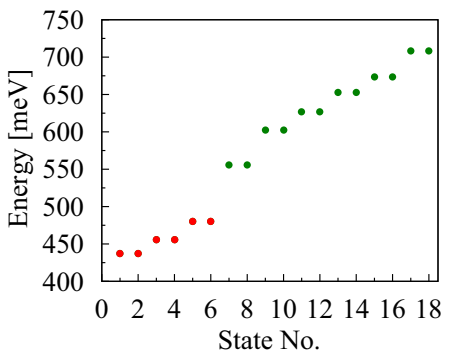

FIG. 7. (Color online) Energy levels for a nonsymmetric triangular ring where the symmetry was broken by increasing the side thickness by $5 \%$ (side parallel to the $y$ axis) and $10 \%$ (subsequent side according to counterclockwise counting). Red points indicate corner-localized states. 


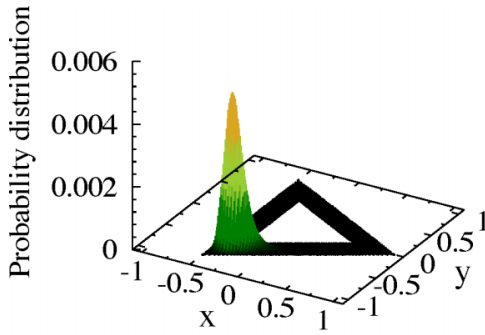

(a) States No. 1, and 2.

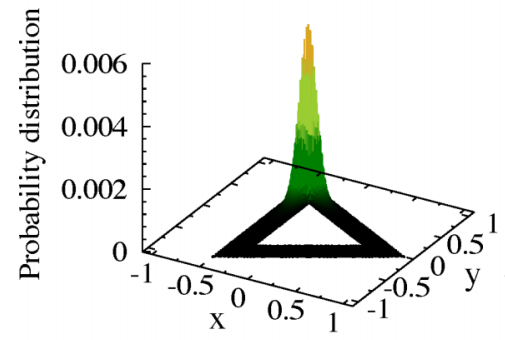

(c) States No. 5, and 6 .

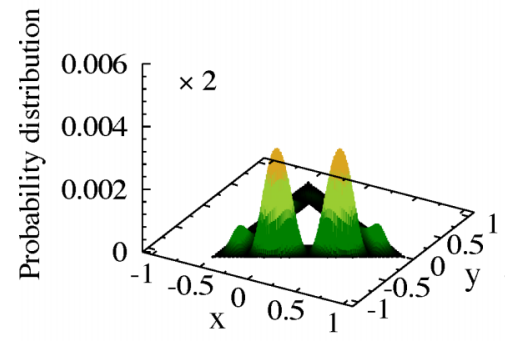

(e) States No. 11, and 12.

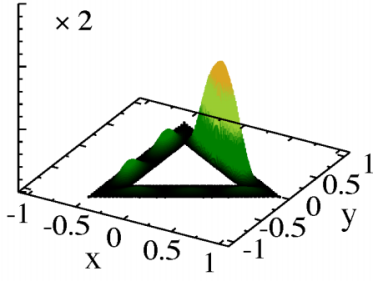

(f) States No. 13, and 14.

FIG. 8. (Color online) Probability distribution for a few chosen energy levels of the nonsymmetric sample shown in Fig. 7. Results shown in (d), (e), and (f) have been graphically magnified by a factor of $2(\times 2)$.

In particular, the ground state is localized in the corner with the largest area [Fig. 8(a)], the two states associated with the second energy level occupy the corner with the medium area [Fig. 8(b)], and the electrons possessing the third energy value may be found in the smallest corner [Fig. 8(c)]. The first state above the corner-localized group is mostly localized in the side region with small probability peaks in corners [Fig. 8(d)]. In general the number of probability peaks increases with the energy. But unlike what happens in a symmetric polygon, one can obtain states with probability distribution concentrated in fewer places than in lower-energy states, as is shown in Figs. 8(e) and 8(f). Similar situations (not shown) occur for rings which are defined by nonsymmetric polygons with uniform thickness, but nonuniform angles. Since the wells formed in corner areas of polygonal rings depend on the angle geometry, they become nonsymmetric when the polygonal sides have different thicknesses. Thus when one side of the polygon is thicker than the others, then the wells formed at its ends have the largest areas and are shifted towards the center of the thicker side. This results in delocalization for the ground-state electrons in those corner areas and shifting them towards the center of the widest side with increasing side thickness. For sufficiently thick sides the wells merge, and the

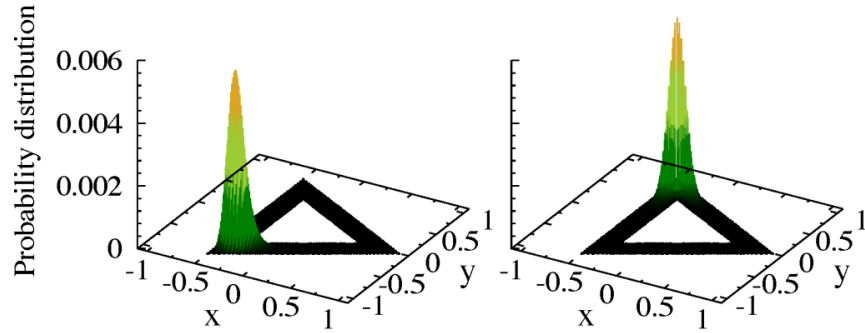

(a) States No. 1, and 2.

(b) States No. 3, and 4.

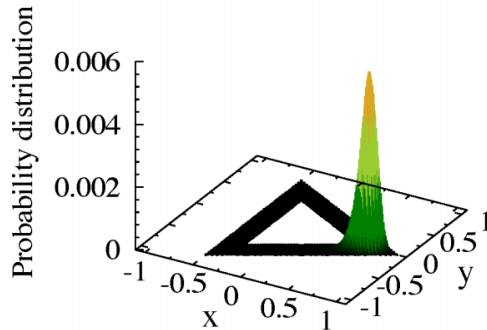

(c) States No. 5, and 6 .

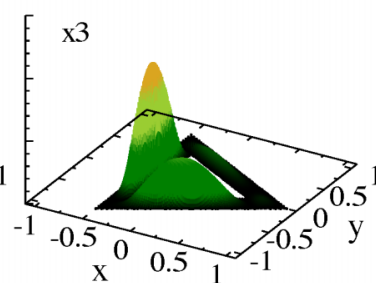

(d) States No. 7, and 8 .

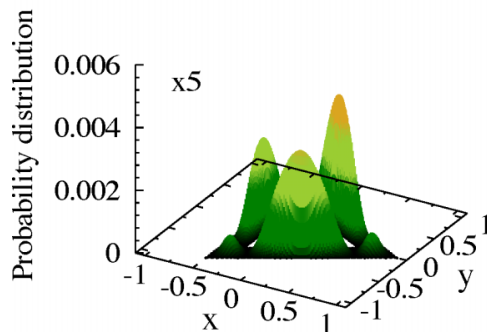

(e) States No. 11, and 12

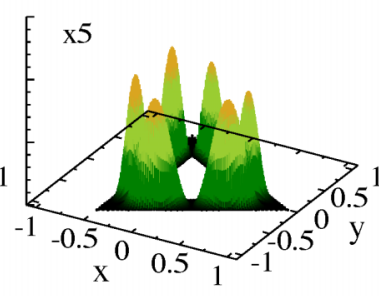

(f) States No. 13, and 14 .
FIG. 9. (Color online) Probability distribution for a few chosen energy levels of a symmetric sample in the presence of an electric field applied along one of the sides $(E \approx 0.11 \mathrm{mV} / \mathrm{nm}, \varphi=\pi / 6)$. Results shown in (d), (e), and (f) have been graphically magnified by a factor of 3 or $5(\times 3, \times 5)$.

ground state becomes localized in the middle of the thicker side, as shown in Ref. [27].

The electron probability density may be controlled externally by applying electric fields. On one hand this may break the symmetric distribution of regular samples; on the other hand it may rebuild, if not perfectly then to some extent, a symmetric distribution in nonsymmetric samples. In Fig. 9 we show electron localization for a geometrically symmetric sample in the presence of an external electric field parallel to one of the sides (forming an angle $\pi / 6$ with the $x$ axis). The electron localization resembles much more that of the nonsymmetric triangle (Fig. 8) than that of the regular one (Fig. 4). But, in contrast to the case with different side thicknesses, here one can change the order of corner occupation. For example, with the electric field rotated such that it becomes parallel to the $y$ axis, the ground state would be localized as in Fig. 9(a), but the corner areas associated with the two higher-energy levels would be reversed with respect to Fig. 9. Or the field perpendicular to one side may localize the ground state in the opposite corner, whereas the two higher (nearly degenerate) energy levels become equally distributed between the remaining two corners. 


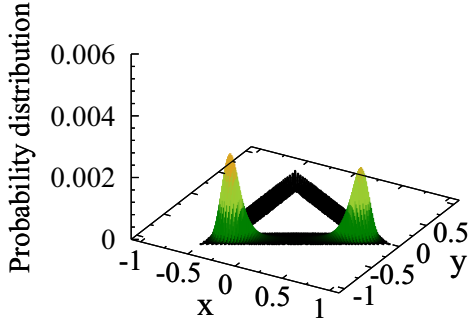

(a) States No. 1, and 2.

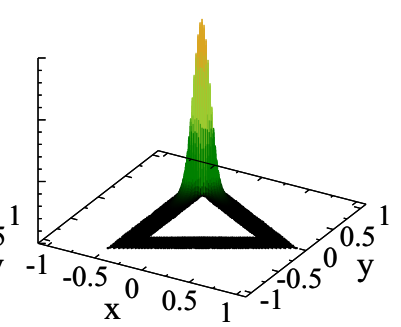

(b) States No. 5, and 6.
FIG. 10. (Color online) Probability distribution associated with the ground state (a) and with the third energy level (b) for a nonsymmetric sample in the presence of an electric field $(E \approx$ $0.67 \mathrm{mV} / \mathrm{nm}, \varphi=\pi / 6)$.

If the previously described sample with different side thicknesses is placed in an external electric field, then it is quite easy to delocalize the ground and the first excited states between the corners at the ends of the widest side [Fig. 10(a)] while the probability distribution of the highest corner-localized state remains localized in the smallest corner [Fig. 10(b)]. Restoration of equal distribution between all three corners is impossible due to the particular shapes and areas of the quantum wells formed in the corners, which strongly differ from each other and cannot be compensated by an external electric field for any angle $\varphi$.

\section{OPTICAL ABSORPTION}

We describe the interaction of electrons in the polygonal rings with an external radiation field in the dipole approximation. The optical absorption coefficient at zero temperature is given by the well-known formula [43-45]

$$
\alpha(\hbar \omega)=A \hbar \omega \sum_{\mathrm{f}}|\langle f|\boldsymbol{\varepsilon} \cdot \boldsymbol{d}| i\rangle|^{2} \delta\left(\hbar \omega-\left(E_{\mathrm{f}}-E_{\mathrm{i}}\right)\right),
$$

with $A$ being a constant containing physical parameters such as the refractive index, the speed of light, the dielectric permittivity, and the sample area, $\varepsilon=(1, \pm i) / \sqrt{2}$ the circular photon polarization, $\boldsymbol{d}$ the dipole moment, and $E_{\mathrm{i}, \mathrm{f}}$ the energies of the initial and final states $|i, f\rangle$, respectively. The dipole matrix elements are

$$
\langle f|\boldsymbol{\varepsilon} \cdot \boldsymbol{d}| i\rangle=\frac{1}{\sqrt{2}} \sum_{q} \Psi^{\dagger}(q, f) \Psi(q, i) r_{q}\left(\cos \phi_{q} \pm i \sin \phi_{q}\right),
$$

where the summation is carried out over all possible basis states $|q\rangle \equiv|k j \sigma\rangle$ and $\Psi(q, a)$ are the amplitudes of the eigenvectors of Hamiltonian (6) in the $q$ basis, $|a\rangle=\sum_{q} \Psi(q, a)|q\rangle$, where $H|a\rangle=E_{a}|a\rangle$. We approximate the $\delta$ function by the spectral weight in the presence of a constant self-energy $\Gamma / 2$,

$$
\delta\left(\hbar \omega-\left(E_{\mathrm{f}}-E_{\mathrm{i}}\right)\right) \approx \frac{\Gamma / 2}{\left[\hbar \omega-\left(E_{\mathrm{f}}-E_{\mathrm{i}}\right)\right]^{2}+(\Gamma / 2)^{2}},
$$

corresponding to a phenomenological broadening of the discrete spectrum of the polygonal ring.

The localization properties discussed in Sec. IV govern the optical absorption through the dipole matrix elements $\langle f|\boldsymbol{\varepsilon} \cdot \boldsymbol{d}| i\rangle$, which depend on the shapes of the wave functions corresponding to states $|i\rangle$ and $|f\rangle$. For simplicity we consider a weak $(0.53 \mathrm{~T})$ magnetic field perpendicular to the ring plane which lifts both degeneracies (due to spin and rotation), but does not considerably affect the electron localization. The chosen magnetic field produces a Zeeman splitting of $0.48 \mathrm{meV}$. Since we do not include a spin-orbit interaction optical transitions may occur only between states with the same spin. We restrict the investigation to two groups of states, corner-localized states and the group consisting of the same number of states above them. For symmetric samples with sharp corners the latter states are purely or mostly side localized [Figs. 4(c) and 4(d) for a triangular sample]. We assume that the system is initially in the ground state, that is, one electron occupies the lowest energy level and we assume a broadening parameter $\Gamma=0.056 \mathrm{meV}$.

In Fig. 11 we compare the absorption spectrum of symmetric triangular [Figs. 11(a) and 11(b)], square [Figs. 11(c) and 11(d)], and hexagonal [Figs. 11(e) and 11(f)] samples with sharp corners. We plot all energy intervals between the ground state and the corner- [(a), (c), and (e)] or side-localized [(b), (d), and (f)] states, respectively, on which we superimpose the optical absorption coefficient calculated according to the formula (9) for an electromagnetic wave circularly polarized in the $x-y$ plane. In principle, for a triangular ring two transitions to the corner-localized and three transitions to the states above the energy gap should be observed. As can be seen, both transitions to the lowest-state domain occur, but each one is coupled with a different orientation of the photon polarization. The reason is that the magnetic field, which points along the positive $z$ direction, creates an orbital splitting of the first two excited states. The lower of them rotates clockwise in the $x$ - $y$ plane, whereas the higher rotates counterclockwise. Although three out of the six states shown in Fig. 11(b) have the same spin as the initial state, only two optical transitions are observed to states which in the absence of a magnetic field would belong to the first, fourfold degenerate energy level above the energy gap. As in the previous case each transition is observed in the presence of only one polarization direction. The same tendency, i.e., coupling of the ground state (twofold degenerate at zero magnetic field) to one of fourfold degenerate states (at zero magnetic field) occurs also for transitions to higher states (not shown). Also, if an electron is initially in a state from the group of fourfold degenerate states then for one polarization orientation it may be excited to the twofold degenerate states and for the other polarization to a fourfold degenerate one. Since for the analyzed triangular sample energy separations between corner-localized states are on the order of tens of meV and the energy distance from the ground state to the side-localized states ranges from 150 to $160 \mathrm{meV}$, thus excitation of the ground state to one of the corner-localized states requires absorption of microwave photons, while transitions to the side-localized states occur in the presence of a near-infrared field. This means that one sample may absorb electromagnetic waves with wavelengths differing by orders of magnitude.

Samples with more corners, in principle, could be expected to allow more transitions because there are more states with the same spin orientation (four for a square and six for a hexagon in each domain). But as shown in Figs. 11(c), 11(d), 11(e), and 11(f), still only two transitions in each state group occur. The absorption coefficient for transitions to 

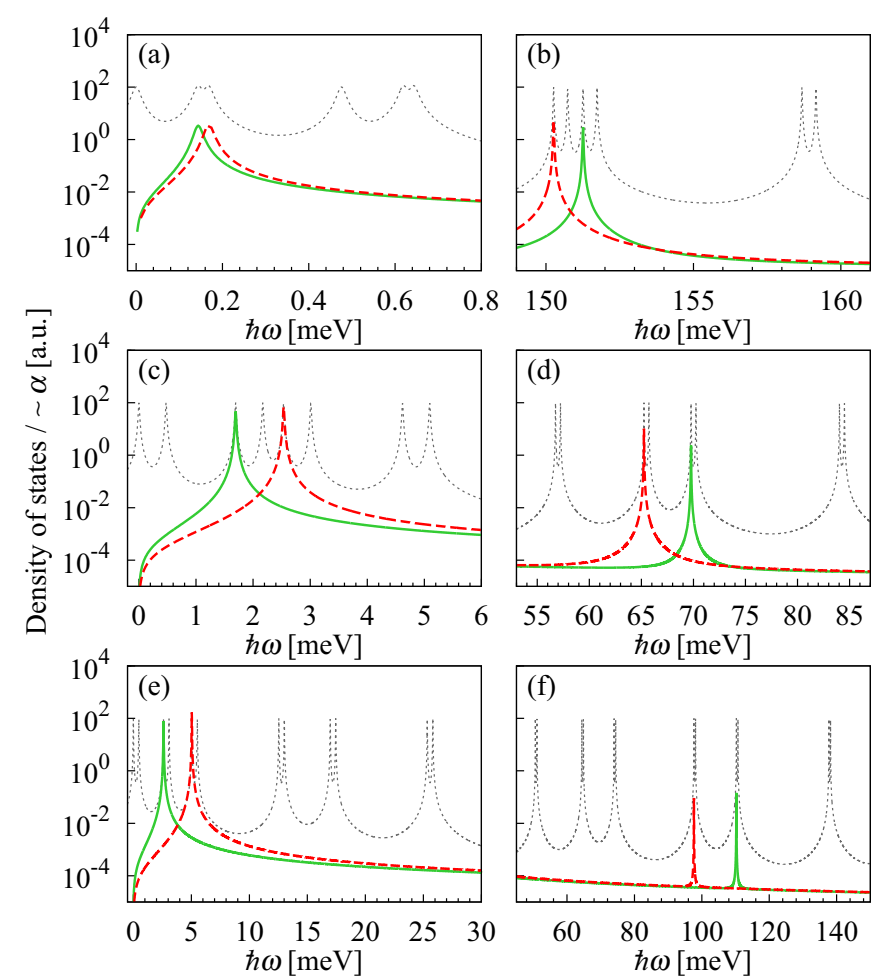

FIG. 11. (Color online) The optically allowed transitions from the ground state in the presence of clockwise (green, solid) and counterclockwise (red, dashed) polarization superimposed on the density of states from Eq. (10) with $\Gamma=0.056 \mathrm{meV}$ (gray, dotted), with the ground-state energy tuned to zero for triangular (a) and (b), square (c) and (d), and hexagonal (e) and (f) symmetric rings with sharp corners in a weak perpendicular magnetic field $(0.53 \mathrm{~T})$. (a), (c), and (e) correspond to transitions to corner-localized states and (b), (d), and (f) transitions to side-localized states. For visibility we use a logarithmic scale for the absorption functions.

corner-localized states increases with the number of corners, while the ratio between its value for transitions to sidelocalized states and transitions to corner-localized states rapidly decreases from over 1 for triangular rings to values on the order of $10^{-3}$ for hexagonal samples. Moreover, the magnitude of the absorption coefficient depends on the polarization type. In the absence of an external electric field it is usually higher for counterclockwise-polarized light. The splitting of the dipole-active absorption peak into mainly two peaks with a growing magnetic field is a well-known phenomena for quantum dots of various shapes [46-48]. The same can be stated about the opposite trends for the evolution of the height of the two absorption peaks with increasing magnetic field.

An external electric field may change the picture, as is shown in Fig. 12 where the field is applied in the ring plane. For both polarization directions all spin-allowed transitions take place, but with different values of the absorption coefficient, which shows that optical experiments may be used to probe the sample geometry. The electric field strength which allows all transitions to be "opened" increases with the number of corners. Triangular samples require weak fields because their corners are relatively well separated, while corners of a
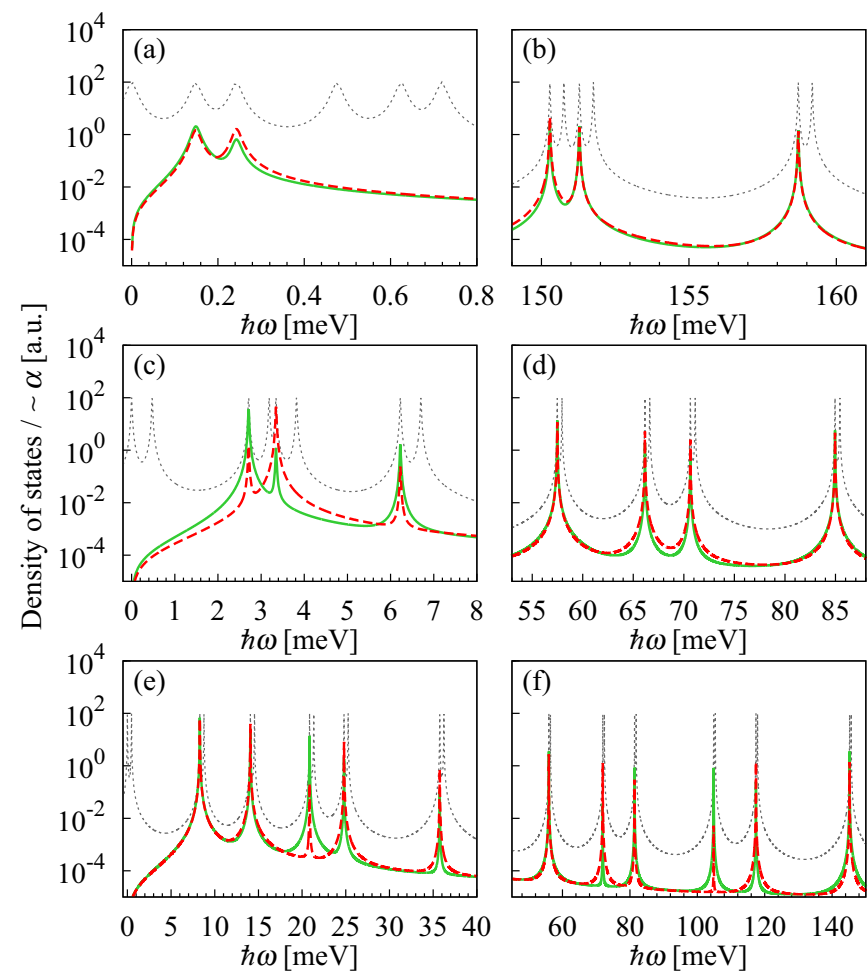

FIG. 12. (Color online) As in Fig. 11 but in the presence of an electric field applied in the ring plane such that it is parallel to one or two ring sides [(a) and (b) $E \approx 0.0056 \mathrm{mV} / \mathrm{nm}, \varphi=\pi / 6$, (c) and (d) $E \approx 0.11 \mathrm{mV} / \mathrm{nm}, \varphi=0$, (e) and (f) $E \approx 0.56 \mathrm{mV} / \mathrm{nm}, \varphi=0]$. For visibility we use a logarithmic scale for the absorption functions.

hexagon defined by the same radius are much closer to each other and thus an electric field of the same value only slightly changes the height of the localization peaks. The dipole matrix elements depend on the symmetry of the wave function and may vanish for some pairs of states, as in the case of transition to the fifth state in Fig. 11(b). But the electric field breaks the wave function symmetry (and shifts the energy levels), and thus it also changes the matrix elements and opens some other transitions, like the one from the ground to the fifth state [Fig. 12(b)]. When the electric field is strong enough to induce localization in single corner areas, then transitions to side-localized states are much more pronounced than those to corner-localized ones. The same situation, for the same symmetry reasons, is observed for the nonsymmetric sample shown in Fig. 8. The absorption spectrum is also sensitive to the angle which the field forms with the $x$ axis; rotation of the field may open, close, or change the strength of some transitions.

\section{CONCLUSIONS}

We studied spectral and optical properties of 2D polygonal quantum rings. We showed that the polygonal geometry induces two- and fourfold degeneracies and formation of an energy gap which depends on the number of corners and the lateral side thickness. In general the lowest-energy states are localized in corner areas, forming a low-energy shell. The probability density is very sensitive to the ring shape. Even if 
the geometry of the sample only slightly differs from a regular ring, the electron localization becomes strongly nonuniform around the polygon. The charge carriers in the ground state are always localized in the corner with the largest area. A certain softening of the corners changes the electron localization only of higher-energy states. The localization pattern may be, to some extent, controlled by an external electric field, which may change the effective potential wells associated with corners. This may also result in breaking the symmetry of regular polygons as inducing a symmetric probability distribution in nonsymmetric samples.

In order to predict basic optical properties related to the corner localization, we calculated the absorption coefficient using the linear response method. We did not include spin-orbit interaction, and thus optical transitions occur only between states with the same spin. Other selection rules are related to the symmetry of the wave functions. Some transitions are forbidden and others are allowed depending on the (circular right or left) photon polarization. In the absence of an external electric field only two transitions from the ground state to the next higher corner-localized states and side-localized states occur. We showed that, as in long triangular core-multishell wires [7-10], triangular rings interact with radiation from different domains, possibly microwave and near-infrared at the same time. Since the external electric field changes the wave function geometry, it also affects the absorption coefficient through the dipole matrix elements and "opens" previously "closed" transitions, blocks others, or changes their intensity, i.e., allows contactless control of optical properties.

\section{ACKNOWLEDGMENTS}

This work was financially supported by the Research Fund of the University of Iceland, the Nordic network NANOCONTROL, project No.: P-13053, and by MINECO-Spain (Grant No. FIS2011-23526).
[1] P. Krogstrup, H. I. Jorgensen, M. Heiss, O. Demichel, J. V. Holm, M. Aagesen, J. Nygard, and A. Fontcuberta i Morral, Nat. Photonics 7, 306 (2013).

[2] R. Peköz, O. B. Malcıŏglu, and J.-Y. Raty, Phys. Rev. B 83, 035317 (2011).

[3] J. Tang, Z. Huo, S. Brittman, H. Gao, and P. Yang, Nature Nanotechnology 6, 568 (2011).

[4] K. Wang, S. C. Rai, J. Marmon, J. Chen, K. Yao, S. Wozny, B. Cao, Y. Yan, Y. Zhang, and W. Zhou, Nanoscale 6, 3679 (2014).

[5] S.-K. Kim, X. Zhang, D. J. Hill, K.-D. Song, J.-S. Park, H.-G. Park, and J. F. Cahoon, Nano Lett. 15, 753 (2015).

[6] Y. Dong, B. Tian, T. J. Kempa, and C. M. Lieber, Nano Lett. 9, 2183 (2009).

[7] S. Gradečak, F. Qian, Y. Li, H.-G. Park, and C. M. Lieber, Appl. Phys. Lett. 87, 173111 (2005).

[8] F. Qian, Y. Li, S. Gradeak, D. Wang, C. J. Barrelet, and C. M. Lieber, Nano Lett. 4, 1975 (2004).

[9] F. Qian, S. Gradeak, Y. Li, C.-Y. Wen, and C. M. Lieber, Nano Lett. 5, 2287 (2005).

[10] F. Qian, Y. Li, S. Gradecak, H.-G. Park, Y. Dong, Y. Ding, Z. L. Wang, and C. M. Lieber, Nat. Mater. 7, 701 (2008).

[11] G. Berkolaiko and P. Kuchment, Introduction to Quantum Graphs (AMS, Providence, RI, 2012).

[12] C. Blömers, T. Rieger, P. Zellekens, F. Haas, M. I. Lepsa, H. Hardtdegen, Ö. Gül, N. Demarina, D. Grützmacher, H. Lüth, and T. Schäpers, Nanotechnology 24, 035203 (2013).

[13] T. Rieger, M. Luysberg, T. Schäpers, D. Grützmacher, and M. I. Lepsa, Nano Lett. 12, 5559 (2012).

[14] F. Haas, K. Sladek, A. Winden, M. von der Ahe, T. E. Weirich, T. Rieger, H. Lüth, D. Grützmacher, T. Schäpers, and H. Hardtdegen, Nanotechnology 24, 085603 (2013).

[15] L. Baird, G. Ang, C. Low, N. Haegel, A. Talin, Q. Li, and G. Wang, in Proceedings of the 25th International Conference on Defects in Semiconductors [Physica B 404, 4933 (2009)].

[16] H. Fan, M. Knez, R. Scholz, K. Nielsch, E. Pippel, D. Hesse, U. Gsele, and M. Zacharias, Nanotechnology 17, 5157 (2006).
[17] H. Shtrikman, R. Popovitz-Biro, A. Kretinin, and M. Heiblum, Nano Lett. 9, 215 (2009).

[18] T. Rieger, D. Grutzmacher, and M. I. Lepsa, Nanoscale 7, 356 (2015).

[19] J. Jadczak, P. Plochocka, A. Mitioglu, I. Breslavetz, M. Royo, A. Bertoni, G. Goldoni, T. Smolenski, P. Kossacki, A. Kretinin, H. Shtrikman, and D. K. Maude, Nano Lett. 14, 2807 (2014).

[20] A. Bertoni, M. Royo, F. Mahawish, and G. Goldoni, Phys. Rev. B 84, 205323 (2011).

[21] M. Royo, A. Bertoni, and G. Goldoni, Phys. Rev. B 87, 115316 (2013).

[22] M. Royo, A. Bertoni, and G. Goldoni, Phys. Rev. B 89, 155416 (2014).

[23] M. Royo, C. Segarra, A. Bertoni, G. Goldoni, and J. Planelles, Phys. Rev. B 91, 115440 (2015).

[24] M. Fickenscher, T. Shi, H. E. Jackson, L. M. Smith, J. M. Yarrison-Rice, C. Zheng, P. Miller, J. Etheridge, B. M. Wong, Q. Gao, S. Deshpande, H. H. Tan, and C. Jagadish, Nano Letters 13, 1016 (2013).

[25] T. Shi, H. E. Jackson, L. M. Smith, N. Jiang, Q. Gao, H. H. Tan, C. Jagadish, C. Zheng, and J. Etheridge, Nano Lett. 15, 1876 (2015).

[26] M.-E. Pistol and C. E. Pryor, Phys. Rev. B 78, 115319 (2008).

[27] A. Ballester, J. Planelles, and A. Bertoni, J. Appl. Phys. 112, 104317 (2012).

[28] C. S. Lent, Appl. Phys. Lett. 56, 2554 (1990).

[29] F. Sols and M. Macucci, Phys. Rev. B 41, 11887 (1990).

[30] D. W. L. Sprung, H. Wu, and J. Martorell, J. Appl. Phys. 71, 515 (1992).

[31] H. Wu, D. W. L. Sprung, and J. Martorell, Phys. Rev. B 45, 11960 (1992).

[32] H. Wu, D. W. L. Sprung, and J. Martorell, J. Appl. Phys. 72, 151 (1992).

[33] H. Wu and D. W. L. Sprung, Phys. Rev. B 47, 1500 (1993).

[34] K. Vacek, A. Okiji, and H. Kasai, Phys. Rev. B 47, 3695 (1993).

[35] H. Xu, Phys. Rev. B 47, 9537 (1993).

[36] C. Estarellas and L. Serra, Superlatt. Microstruct. 83, 184 (2015). 
[37] A. Ballester, C. Segarra, A. Bertoni, and J. Planelles, EPL (Europhys. Lett.) 104, 67004 (2013).

[38] C. Daday, A. Manolescu, D. C. Marinescu, and V. Gudmundsson, Phys. Rev. B 84, 115311 (2011).

[39] B. M. Wong, F. Léonard, Q. Li, and G. T. Wang, Nano Lett. 11, 3074 (2011).

[40] A. Fuhrer, S. Luscher, T. Ihn, T. Heinzel, K. Ensslin, W. Wegscheider, and M. Bichler, Nature (London) 413, 822 (2001).

[41] M. Aichinger, S. A. Chin, E. Krotscheck, and E. Räsänen, Phys. Rev. B 73, 195310 (2006).

[42] M. Niţă, D. C. Marinescu, A. Manolescu, and V. Gudmundsson, Phys. Rev. B 83, 155427 (2011).
[43] H. Haug and S. W. Koch, Quantum Theory of the Optical and Electronic Properties of Semiconductors, 5th ed. (World Scientific, Singapore, 2009).

[44] S. L. Chuang, Physics of Optoelectronic Devices (John Wiley and Sons, New York, 1995).

[45] H. Hu, J.-L. Zhu, and J.-J. Xiong, Phys. Rev. B 62, 16777 (2000).

[46] V. Gudmundsson and R. R. Gerhardts, Phys. Rev. B 43, 12098 (1991).

[47] R. Krahne, V. Gudmundsson, C. Heyn, and D. Heitmann, Phys. Rev. B 63, 195303 (2001).

[48] I. Magnúsdóttir and V. Gudmundsson, Phys. Rev. B 60, 16591 (1999). 